\title{
An efficient, multiple range random walk algorithm to calculate the density of states
}

\author{
Fugao Wang and D. P. Landau \\ Center for Simulational Physics, The University of Georgia, Athens, Georgia 30602
}

(February 1, 2008)

\begin{abstract}
We present a new Monte Carlo algorithm that produces results of high accuracy with reduced simulational effort. Independent random walks are performed (concurrently or serially) in different, restricted ranges of energy, and the resultant density of states is modified continuously to produce locally flat histograms. This method permits us to directly access the free energy and entropy, is independent of temperature, and is efficient for the study of both 1st order and 2nd order phase transitions. It should also be useful for the study of complex systems with a rough energy landscape.

64.60.Cn, 05.50.+q, 02.70.Lq
\end{abstract}

Computer simulation has become an essential tool in condensed matter physics [1], particularly for the study of phase transitions and critical phenomena. The workhorse for the past half-century has been the Metropolis importance sampling algorithm, but more recently new, efficient algorithms have begun to play a role in allowing simulation to achieve the resolution which is needed to accurately locate and characterize phase transitions. For example, cluster flip algorithms, beginning with the seminal work of Swendsen and Wang [2], have been used to reduce critical slowing down near 2 nd order transitions. Similarly, the multicanonical ensemble method [3] was introduced to overcome the tunneling barrier between coexisting phases at 1st order transitions, and this approach also has utility for systems with a rough energy landscape [4] 6 . In both situations, histogram reweighting techniques [7] can be applied in the analysis to increase the amount of information that can be gleaned from simulational data, but the applicability of reweighting is severely limited in large systems by the statistical quality of the "wings" of the histogram. This latter effect is quite important in systems with competing interactions for which short range order effects might occur over very broad temperature ranges or even give rise to frustration that produces a very complicated energy landscape and limit the efficiency of other methods.

In this paper, we introduce a new, general, efficient Monte Carlo algorithm that offers substantial advantages over existing approaches. Unlike conventional Monte Carlo methods that directly generate a canonical distribution at a given temperature $g(E) e^{-E / K_{\mathrm{B}} T}$, our approach is to estimate the density of states $g(E)$ accurately via a random walk which produces a flat histogram in energy space. The method can be further enhanced by performing multiple random walks, each for a different range of energy, either serially or in parallel fashion. The resultant pieces of the density of states can be joined together and used to produce canonical averages for the calculation of thermodynamic quantities at essentially any temperature. We will apply our algorithm to the 2-dim ten state Potts model and Ising model which have 1st- and 2nd-order phase transitions, respectively, to demonstrate the efficiency and accuracy of the method.

Our algorithm is based on the observation that if we perform a random walk in energy space with a probability proportional to the reciprocal of the density of states $\frac{1}{g(E)}$, then a flat histogram is generated for the energy distribution. This is accomplished by modifying the estimated density of states in a systematic way to produce a "flat" histogram over the allowed range of energy and simultaneously making the density of states converge to the true value. At the very beginning of the random walk, the density of states is a priori unknown, so we simply set all densities of states $g(E)$ for all energies $E$ to $g(E)=1$. Then we begin our random walk in energy space by flipping spins randomly. In general, if $E_{1}$ and $E_{2}$ are energies before and after a spin is flipped, the transition probability from energy level $E_{1}$ to $E_{2}$ is simply:

$$
p\left(E_{1} \rightarrow E_{2}\right)=\min \left(\frac{g\left(E_{1}\right)}{g\left(E_{2}\right)}, 1\right)
$$

This is also the probability to flip the spin. Each time an energy level $E$ is visited, we update the corresponding density of states by multiplying the existing value by a modification factor $f>1$, i.e. $g(E) \rightarrow g(E) * f$. The initial modification factor can be as large as $f=f_{0}=e^{1} \simeq$ $2.71828 \ldots$ which allows us to reach all possible energy levels very quickly, even for large systems. We keep walking randomly in energy space and modifying the density of states until the accumulated histogram $H(E)$ is "flat". At this point, the density of states converges to the true value with an accuracy proportion to $\ln (f)$. We then reduce the modification factor to a finer one according to some recipe like $f_{1}=\sqrt{f_{0}}$ (any function that monotonically decreases to 1 will do) and reset the histogram $H(E)=0$. Then we begin the next level random walk with a finer modification factor $f=f_{1}$, continuing until the histogram is again "flat" after which we stop and reduce the modification factor as before, i.e. $f_{i+1}=\sqrt{f_{i}}$. We stop the simulation process when the modification factor is smaller than some predefined final value (such as $\left.f_{\text {final }}=\exp \left(10^{-8}\right) \simeq 1.00000001\right)$. It is very clear that the modification factor $f$ in our random walk acts 
as a control parameter for the accuracy of the density of states during the simulation and also determines how many MC sweeps are necessary for the whole simulation. It is impossible to obtain a perfectly flat histogram and the phrase "flat histogram" in this paper means that histogram $H(E)$ for all possible $E$ is not less than $80 \%$ of the average histogram $\langle H(E)\rangle$. Since the density of states is modified every time the state is visited, we only obtain a relative density of states at the end of the simulation. To calculate the absolute values, we use the condition that the number of ground states for the Ising model is 2 (all spins are up or down) to re-scale the density of states; and if multiple walks are performed within different energy ranges, they must be matched up at the boundaries in energy.

Because of the exponential growth of the density of states in energy space, it is not efficient to simply update the density of states until enough histogram entries are accumulated. All methods based on the accumulation of entries, such as the histogram method [7], Lee's version of the multicanonical method (entropic sampling) [3], the broad histogram method [8] and the flat histogram method [9,10] have the problem of scalability for large systems. These methods suffer from systematic errors and substantial deviations which increase rapidly for large system size. The algorithm proposed in this paper is of both high efficiency and accuracy over wide ranges of temperature for sizes that are beyond those that are tractable by other approaches.

We should point out here that during the random walk (especially for the early stage of iteration), the algorithm does not exactly satisfy the detailed balance condition, since the density of states is modified constantly during the random walk in energy space; however, after many iterations, the density of states converges to the true value very quickly as the modification factor approaches 1. From eq. (1), we have:

$$
\frac{1}{g\left(E_{1}\right)} p\left(E_{1} \rightarrow E_{2}\right)=\frac{1}{g\left(E_{2}\right)} p\left(E_{2} \rightarrow E_{1}\right)
$$

where $\frac{1}{g\left(E_{1}\right)}$ is the probability at the energy level $E_{1}$ and $p\left(E_{1} \rightarrow E_{2}\right)$ is the transition probability from $E_{1}$ to $E_{2}$ for the random walk. We can thus conclude that the detailed balance condition is satisfied to within the accuracy proportion to $\ln (f)$.

The convergence and accuracy of our algorithm may be tested for a system with a 2 nd order transition, the $L \times L$ Ising square lattice with nearest neighbor coupling which is generally perceived as an ideal benchmark for new theories [11] and simulation algorithms [7, 12]. We simulated both small lattices for which exact results are available as well as $L=256$ for which exact enumeration is impossible. In Fig. 1, the densities of states estimated by our algorithm are shown along with the exact results obtained by the method proposed by Beale 13]. We only show the density for systems up to $L=50$ which is the maximum size we can calculate with the Mathematica program used in the reference 113. Since no difference is visible, we show the relative error $\varepsilon(\log (g(E)))$, which is defined as $\varepsilon(X) \equiv\left|\left(X_{\text {sim }}-X_{\text {exact }}\right) / X_{\text {exact }}\right|$ for a general quantity $X$ in this paper. With our algorithm we obtain an average error as small as $0.035 \%$ on the $32 \times 32$ lattice with $7 \times 10^{5}$ sweeps. It is possible to estimate the density of states for small systems with the broad histogram method [8]. Recent broad histogram simulational data [14] for the $2 \mathrm{D}$ Ising model on a $32 \times 32$ lattice with $10^{6} \mathrm{MC}$ sweeps yielded an average deviation of the microcanonical entropy from about $0.08 \%$ from the exact solution 13.

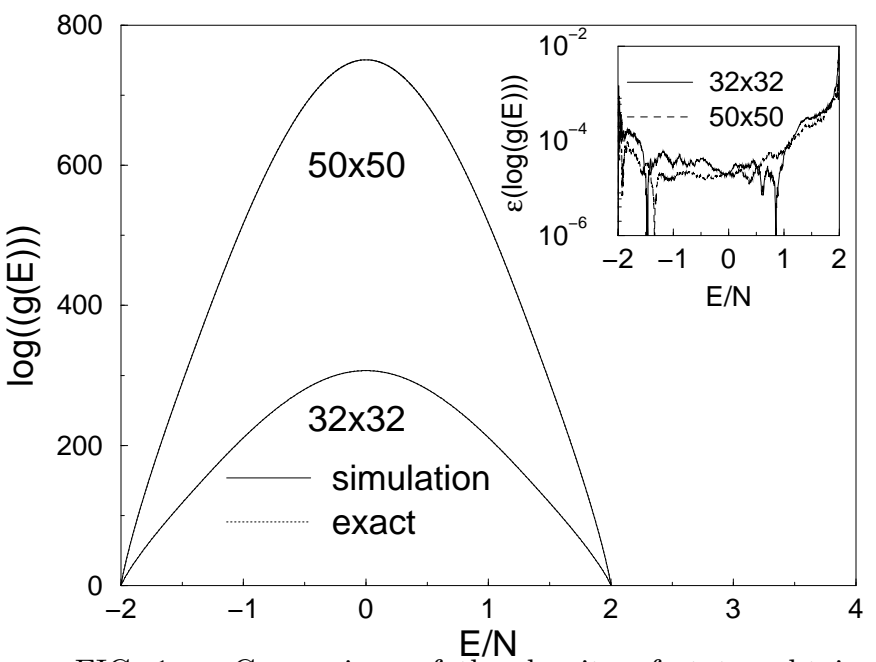

FIG. 1. Comparison of the density of states obtained by our algorithm for $2 \mathrm{D}$ Ising model and the exact results calculated by the method in reference [13]. Relative errors $(\varepsilon(\log (g(E)))$ are shown in the inset.

With the Monte Carlo algorithm proposed in this paper, we can estimate the density of states efficiently even for large systems. Because of the symmetry of the density of states for Ising model $g(E)=g(-E)$, we only need to estimate the density of states in the region $E / N \in[-2,0]$, where $N$ is total lattice sites. To speed up the simulation for $L=256$, we perform 15 independent random walks, each for a different region of energy from $E / N=-2$ to $E / N=0.2$ using $f_{\text {final }}=\exp \left(10^{-8}\right)$. To reduce the "boundary effect", random walks over adjacent energy regions overlap by $\Delta E / N=0.06$. The density of states for $E / N \in[-2,0.2]$ is obtained by joining 15 densities of states from random walks on different energy regions using a total simulational effort of only $6.1 \times 10^{6} \mathrm{MC}$ sweeps.

One advantage of our algorithm is that we can readily calculate the Gibbs free energy and the entropy, quantities which are not directly available in conventional Monte Carlo simulations. With the density of states, the Gibbs free energy can be calculated by 


$$
F(T)=-k_{\mathrm{B}} T \ln (Z)=-k_{\mathrm{B}} T \ln \left(\sum_{E} g(E) e^{-\beta E}\right) .
$$

Although it is impossible to calculate the exact density of states of Ising model on a lattice as large as $L=256$ with the method proposed by Beale [13], the free energy and specific heat were calculated exactly by Ferdinand and Fisher [15] on finite-size lattices. In Fig. 2, we compare simulational data and exact solutions for the Gibbs free energy as a function of temperature. The agreement is excellent and a more stringent test of the accuracy shows that the relative error $\varepsilon(F)$ is smaller than $0.0008 \%$ for temperature region $T \in[0,8]$.

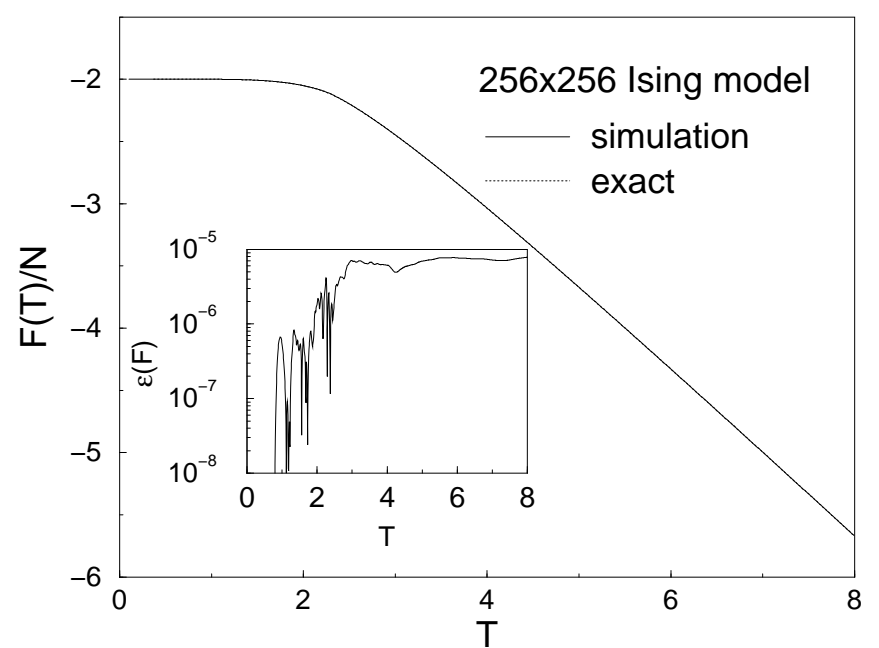

FIG. 2. Comparison of the Gibbs free energy per lattice site calculated directly from the density of states from our simulation for the $L=256$ Ising model and the exact solutions from reference [15]. The relative errors $\varepsilon(F)$ are shown in the inset. The density of states was obtained by random walks with only $6.1 \times 10^{6} \mathrm{MC}$ sweeps totally.

The entropy is a very important thermodynamic quantity that cannot be calculated directly in conventional Monte Carlo simulations. It can be estimated by integrating over other thermodynamic quantities, such as specific heat, but such calculations are not so reliable since the specific heat itself is not so easy to estimate accurately. With an accurate density of states estimated by our method, the entropy can be calculated easily by $S(T)=\frac{U(T)-F(T)}{T}$ where $U(T)=$ $\langle E\rangle_{T} \equiv \sum_{E} E g(E) e^{-\beta E} / \sum_{E} g(E) e^{-\beta E}$ is the internal energy. According to our calculation, the errors for $L=$ 256 are smaller than $1.2 \%$ in all temperature region $T \in[0,8]$. 16] Very recently, with the flat histogram method [9] and the broad histogram method [8], the entropy was estimated with $10^{7} \mathrm{MC}$ sweeps for the same model on $32 \times 32$ lattice; however, the errors in reference [10] are even much bigger than our errors for $256 \times 256$ !

A more stringent test of the accuracy of the density of states is calculation of the specific heat defined by the fluctuation expression:

$$
C(T)=\frac{\left\langle E^{2}\right\rangle_{T}-\langle E\rangle_{T}{ }^{2}}{T^{2}}
$$

Our simulational data on the finite-size lattice are compared with the exact solution obtained by Ferdinand and Fisher 15 in Fig.3. A stringent test of the accuracy is provided by the inset which shows the relative error $\varepsilon(C)$. The average error over the entire range $T \in[0.4,8]$ only used a total of $6.1 \times 10^{6}$ MC sweeps is $0.39 \%$. The relative errors are not bigger than $4.5 \%$ even with fine scale near $T_{\mathrm{c}}$. Recently, Wang, Tay and Swendsen [12] estimated the specific heat of the same model on a $64 \times 64$ lattice by the transition matrix Monte Carlo re-weighting method [17], and for a simulation with $2.5 \times 10^{7} \mathrm{MC}$ sweeps, the maximum error in temperature region $T \in[0,8]$ was about $1 \%$. When we apply our algorithm to the same model on the $64 \times 64$ lattice, with a final modification factor of 1.000000001 and a total of $2 \times 10^{7} \mathrm{MC}$ sweeps on single processor, the errors of the specific heat are reduced below $0.7 \%$ for all temperature [16]. The relatively large errors at low temperature reflect the small values for the specific heat at low temperature. The errors in specific heat estimated from the density of states with broad histogram method are obviously visible even for systems as small as $32 \times 32$ [8] whereas with our method, such differences are invisible even for a system as large as $256 \times 256$.

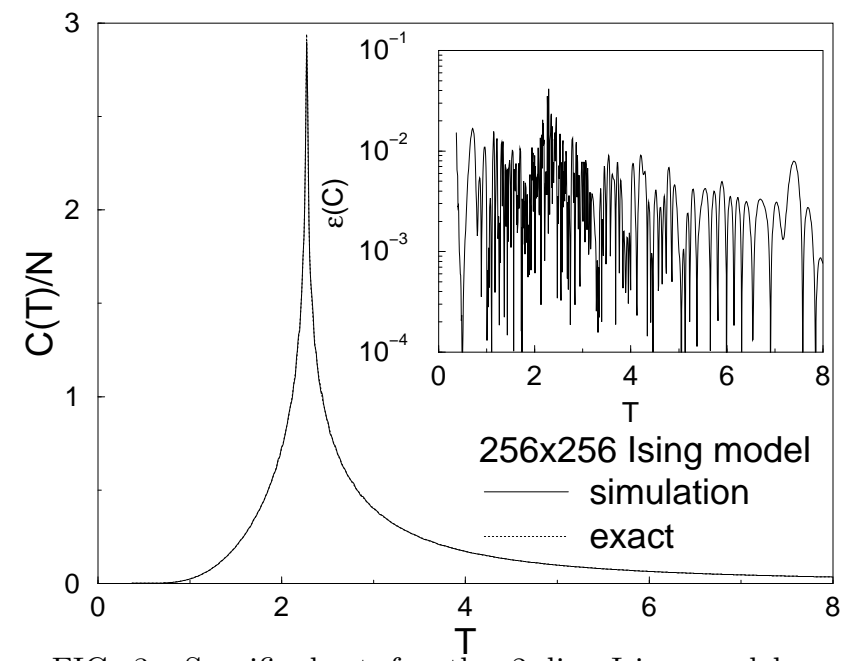

FIG. 3. Specific heat for the 2-dim Ising model on a $256 \times 256$ lattice in a wide temperature region. The relative error $\epsilon(C)$ are shown in the inset in the figure.

With our algorithm, we not only dramatically reduce the computational effort by avoiding multiple simulations for different temperatures close to the transition, but also overcome the slow kinetics at low temperature or near $T_{c}$ for both first-order and second-order phase transitions since the random walk does not depend on the temperature. To show how our simulation method overcomes the tunneling barrier between order and disorder phases at a 1st-order phase transition, we perform random walks 
to calculate the density of states for the $2 \mathrm{D}$ ten state Potts model [18] with nearest neighbor interactions on square lattices of size $60 \leq L \leq 200$. In Fig.4, we show the canonical distributions at the temperatures at which the peaks are of equal height. Because of the double peak structure of strongly 1st-order phase transitions [19], conventional Monte Carlo simulations are not efficient since it takes an extremely long time to tunnel from one peak to the other. Considering the valley which we find for $L=200$ is as deep as $9 \times 10^{-10}$, it is impossible for conventional Monte Carlo algorithms to overcome such a tunneling barrier with available computational resources.

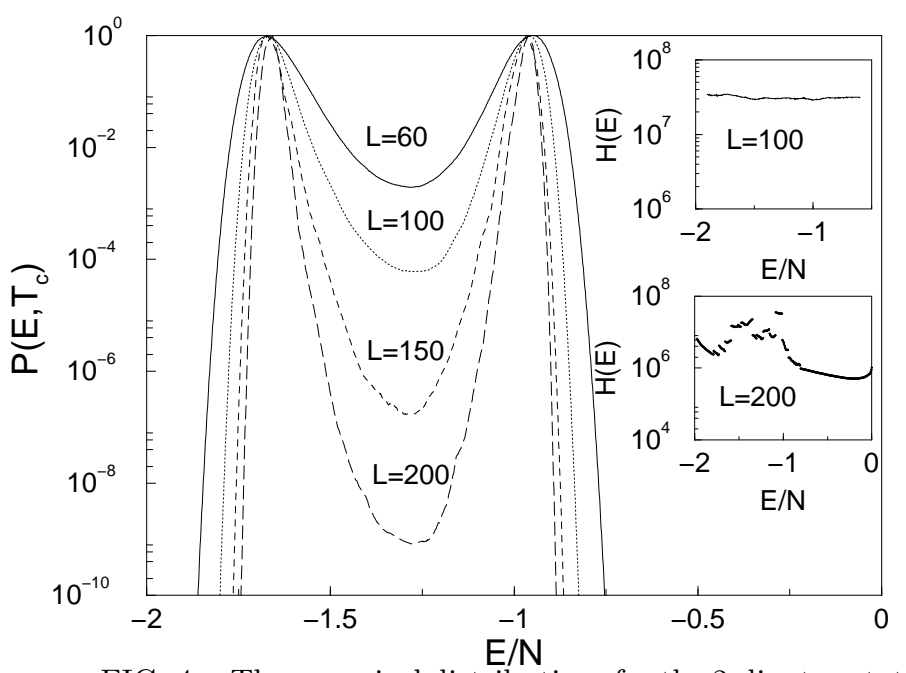

FIG. 4. The canonical distributions for the 2-dim ten state Potts model on $L \times L$ lattice at the transition temperature $P\left(E, T_{\mathrm{c}}\right) \equiv g(E) e^{-E / K_{\mathrm{B}} T_{\mathrm{c}}}$. For $L=150$ and 200, multiple random walks were performed in different energy regions with locally flat histograms. The distributions at peaks are normalized to 1 . The transition temperature $T_{\mathrm{c}}(L)$ is 0.701243 for $L=200 ; T_{\mathrm{c}}(\infty)=0.701232 \ldots$ (exact solution). In the inset, we show the overall histograms of the random walks for $L=100$ and 200 . $3.1 \times 10^{7}$ visits per energy level were used for $L=100$ with a single random walk. With multiple random walks, the density of states for $L=200$ was obtained with only $9.8 \times 10^{6}$ visits per energy level.

All thermodynamic quantities we discussed so far are directly related to energy. It is also possible to calculate any quantities which may not directly relate to energy. [16] As an example, the order-parameter for the 2D Ising model can be calculated by $|M(T)|=$ $\sum_{E}|M(E)| g(E) e^{-\beta E} / \sum_{E} g(E) e^{-\beta E}$ where $M(E)$ is the average value of the order-parameter at energy level $E$ during the random walk. The random walk is not restricted to energy space, and our algorithm can be applied to any other parameter space. To apply our algorithm to a new system, the only thing we need to know is the Hamiltonian. The algorithm can be optimized to estimate the relevant density of states to the property and temperature range of interest. This new Monte Carlo algorithm should be extremely useful for the study of the complex systems such as spin glass models 5 and protein folding problems [6] where the energy landscape is very rough and where it is already known that there are problems with other optimization algorithms.

We would like to thank C. K. Hu, N. Hatano, P. D. Beale, S. P. Lewis and H-B Schuttler for helpful comments and suggestions. The research project was supported by the National Science Foundation under Grant No. DMR-9727714.

[1] D. P. Landau and K. Binder, A Guide to Monte Carlo Methods in Statistical Physics, (Cambridge U. Press, Cambridge, 2000).

[2] R. H. Swendsen and J.-S. Wang, Phys. Rev. Lett. 58, 86 (1987), U. Wolff, Phys. Rev. Lett. 62, 361 (1989).

[3] B. A. Berg and T. Neuhaus, Phys. Rev. Lett. 68, 9 (1992), Phys. Lett. B 267, 249 (1991), J. Lee, Phys. Rev. Lett. 71 , 211 (1993). B. A. Berg J. Stat. Phys. 82, 323 (1996), B. A. Berg, Nucl. Phys. B, 63, 982 (1998).

[4] W. Janke and S. Kappler, Phys. Rev. Lett. 74, 212 (1995).

[5] B. A. Berg and T. Celik, Phys. Rev. Lett. 69, 2292 (1992), B. A. Berg and W. Janke, Phys. Rev. Lett. 80, 4771 (1998), N. Hatano and J. E. Gubernatis in Computer Simulation Studies in Condensed Matter Physics XII, D. P. Landau, S. P. Lewis and H.-B. Schuttler (eds) (Springer, Berlin, Heidelberg, 2000).

[6] N. S. Alves and U. Hansmann, Phys. Rev. Lett. 84, 1836 (2000).

[7] A. M. Ferrenberg and R. H. Swendsen, Phys. Rev. Lett. 61, 2635 (1988), 63, 1195 (1989).

[8] P. M. C. de Oliveira, T. J. P. Penna and H. J. Herrmann, Eur. Phys. J. B. 1, 205 (1998), P. M. C. de Oliveira, Eur. Phys. J. B. 6, 111 (1998).

[9] Jian-Sheng Wang, Eur. Phys. J. B. 8, 287 (1998), Condmatt-9909177.

[10] Jian-Sheng Wang and Lik Wee Lee, Comput. Phys. Commun. 127, 131, (2000).

[11] D. P. Landau, Phys Rev. B 13, 2997 (1976).

[12] Jian-Sheng Wang, T. K. Tay and R. H. Swendsen, Phys. Rev. Lett. 82, 476 (1999).

[13] P. D. Beale, Phys. Rev. Lett. 76, 78 (1996).

[14] A. R. Lima, P. M. C. de Oliveira and T. J. P. Penna, J. Stat. Phys. 99, 691, (2000).

[15] A. E. Ferdinand and M. E. Fisher, Phys. Rev. 185, 832 (1969).

[16] Fugao Wang and D. P. Landau, unpublished data.

[17] R. H. Swendsen, J. S. Wang, S. T Li, B. Diggs, C. Genovese and J. B. Kadane, Cond-matt-9908461.

[18] F. Y. Wu, Rev. Mod. Phys. 54, 235 (1982).

[19] M. S. S. Challa, D. P. Landau and K. Binder Phys. Rev. B 34, 1841, (1986). 